Revue des patrimoines

43 | 2021

Des écoles d'art académiques aux écoles d'art : des collections et des lieux, un patrimoine à valoriser

\title{
L'Accademia Albertina di Belle Arti di Torino
}

Le sue origini, la sua storia, le sue collezioni

\section{Marco Carassi}

\section{(2) OpenEdition}

\section{Journals}

Edizione digitale

URL: http://journals.openedition.org/insitu/29321

DOI: $10.4000 /$ insitu. 29321

ISSN: 1630-7305

\section{Editore}

Ministère de la Culture

Notizia bibliografica digitale

Marco Carassi, «L'Accademia Albertina di Belle Arti di Torino », In Situ [En ligne], 43 | 2021, document 13, mis en ligne le 12 janvier 2021, consulté le 14 janvier 2021. URL : http://journals.openedition.org/ insitu/29321 ; DOI : https://doi.org/10.4000/insitu.29321

Questo documento è stato generato automaticamente il 14 janvier 2021.

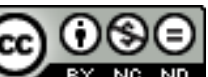

In Situ Revues des patrimoines est mis à disposition selon les termes de la licence Creative Commons Attribution - Pas d'Utilisation Commerciale - Pas de Modification 4.0 International. 


\section{L'Accademia Albertina di Belle Arti di Torino}

Le sue origini, la sua storia, le sue collezioni

\section{Marco Carassi}

1 Il 29 agosto 1678 Maria Giovanna Battista di Savoia-Nemours, reggente degli Stati della Casa di Savoia dopo la morte del duca Carlo Emanuele II, institutionalizza un'antica corporazione di mestiere creando l'Accademia dei pittori, degli scultori e degli architetti di Torino ${ }^{1}$. La prima collezione di opere d'arte destinate alla didattica è formata dalle donazioni cui sono tenuti gli accademici, uomini e donne. Sotto Vittorio Amedeo II, nel 1716, sono approvati gli statuti ispirati a quelli dell'Accademia romana di San Luca².

2 Nel 1778 l'Accademia è rifondata per iniziativa del conte Roberto Malines di Bruino, molto critico dello stato in cui si era ridotta l'istituzione dopo un secolo di vita "il ne manquoit que peu de chose à l'école de peinture: un maitre et des élèves ${ }^{3}$ ". La direzione affidata in tale occasione al pittore francese di formazione romana Laurent Pécheux, è caratterizzata da stretti legami con le accademie di Roma, Parma e Parigi. A lui si deve la formazione di una gipsoteca mediante l'acquisto a Roma di copie di statue antiche, di cui si conosce un elenco di 24 gessi datato 1811. Pécheux nomina Carlo Antonio Porporati responsabile della scuola di incisione, Giuseppe Duprà della scuola di pittura, Ignazio et Filippo Collino della scuola di scultura ${ }^{4}$.

3 Joseph-Jérôme Lefrançois de Lalande, nel suo Voyage en Italie (1786), descrive la scuola e il suo corpo insegnante ${ }^{5}$. All'época napoleonica, quando il Piemonte è annesso alla Francia, l'Accademia di Torino è integrata nel sistema universitario imperiale. nel 1808 la Scuola speciale delle arti del disegno di Torino è collegata all'Accademia di Parigi ${ }^{6}$. I corsi sono tenuti in città, in luoghi diversi, da parte di insegnanti famosi come lo scultore Giacomo Spalla e l'architetto Ferdinando Bonsignore. La "petite bibliothèque" di cui parla Pécheux per l'istruzione degli allievi comprende la Bibbia, le opere di Omero, Virgilio e Ovidio, molti trattati di storia antica e moderna, biografie di artisti, repertori di iconologia per la pittura e la scultura [fig. ${ }^{\circ} 1$ ]. Donazioni di libri, incisioni e litografie si succedono secondo le tendenze artistiche via via emergenti. Per un periodo 
limitato, un Museo didattico di sculture e pitture è istituito nel palazzo imperiale (ex reale) di Torino, mentre in città, in occasione dei passaggi di Napoleone, si organizzano esposizioni di Objets d'art, manufactures et métiers. Le esposizioni annuali degli allievi dell'Accademia sono collocate nell'antico convento di san Francesco da Paola ${ }^{7}$.

Fig. 1

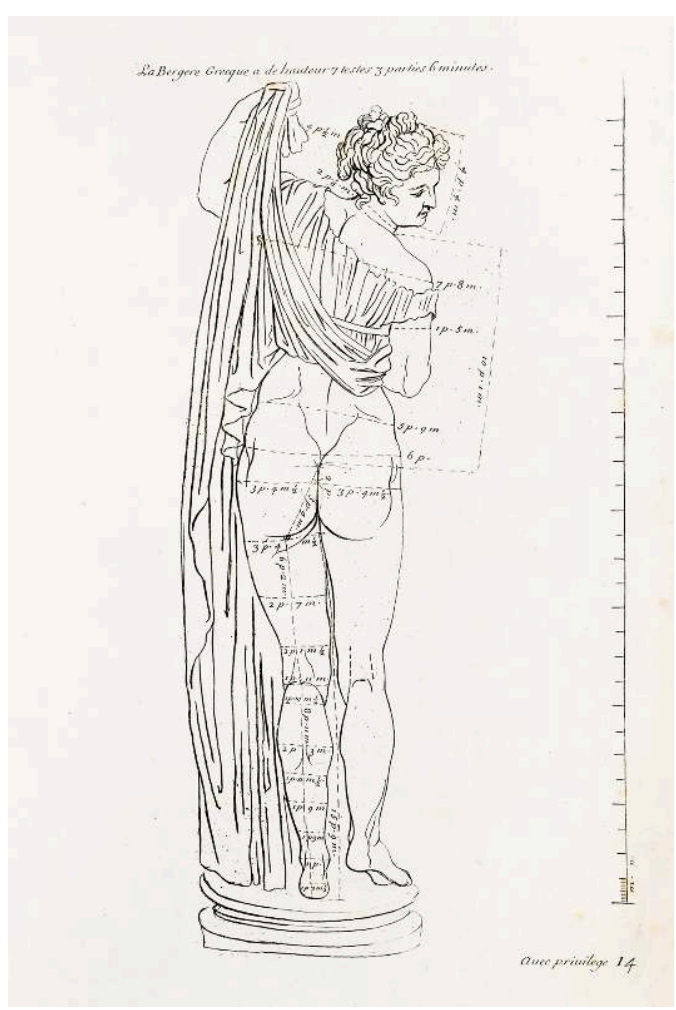

AUDRAN Gérard, Les Proportions du corps humain mesurées sur les plus belles figures de l'Antiquité, Parigi, Joubert, 1801 [1683], planche 14 : La Bergère grecque, ossia Vénus callipigia. L'immagine porta delle misure utili agli scultori.

4 Nel 1820 il re Carlo Felice rimette in vigore il regolamento del 1778 e nomina segretario perpetuo dell'Accademia il pittore nizzardo Giovanni Battista Biscarra, che esercita una profonda influenza fino al $1851^{8}$. Il re Carlo Alberto nel 1833 decide l'installazione definitiva dell'Accademia nell'antico convento di san Francesco da Paola, dove tutt'ora si trova [fig. $\left.{ }^{\circ} 2\right]^{9} \mathrm{e}$ le fa dono nel 1837 dell'opera di Winkelmann sull'arte del disegno tra gli antichi ${ }^{10}$. 
Fig. 2

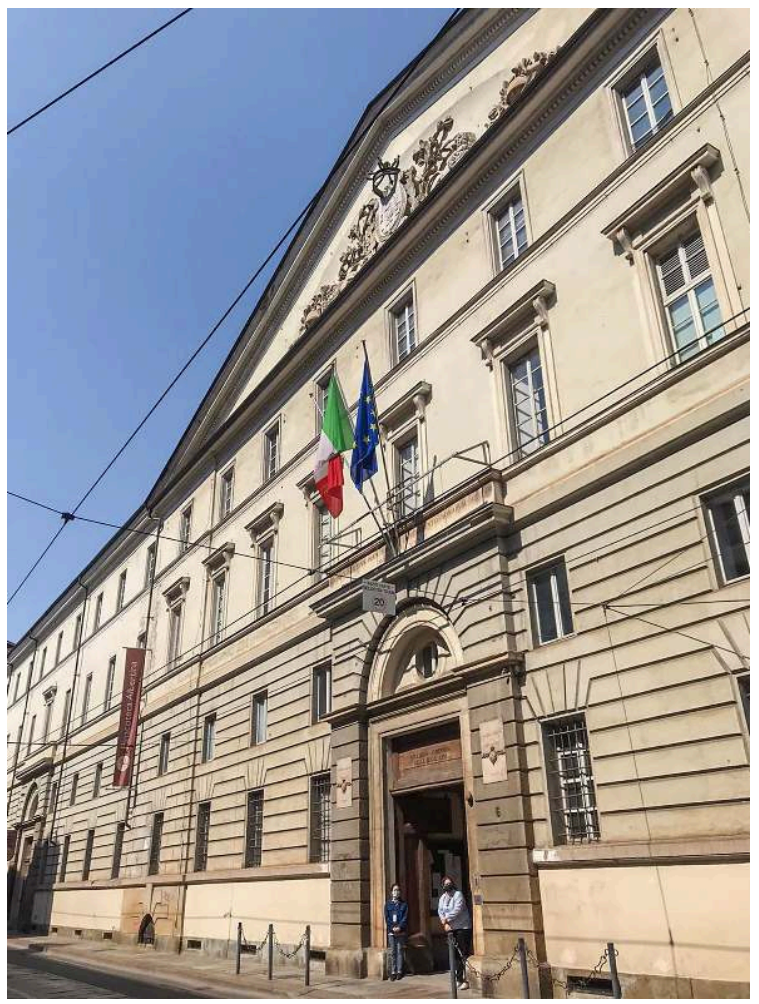

Accademia Albertina, facciata principale.

(c) foto Fabio Amerio (Accademia Albertina di Belle Arti, Torino).

5 L'architettura dell'edificio, nato nel XVII ${ }^{\circ}$ secolo, è adattato a più riprese: a partire dal 1822 dall'architetto Giuseppe Maria Talucchi (cui è dovuta la nuova facciata monumentale e l'imponente "Rotonda" collocata nel grande cortile [fig. ${ }^{\circ}$ ], dopo il 1834 dagli architetti Carlo Bernardo Mosca ed Ernesto Melano (l'ingresso munito di colonne, la sala del re Carlo Alberto e lo scalone monumentale [fig. ${ }^{\circ} 4$; fig. ${ }^{\circ 5}$ ], dopo il $1856 \mathrm{su}$ richiesta dello scultore Vincenzo Vela per l'ingrandimento del suo studio-atelier, e sopratutto con i lavori divenuti indispensabili dopo i gravi danni dei bombardamenti del $1944^{11}$. 
Fig. 3

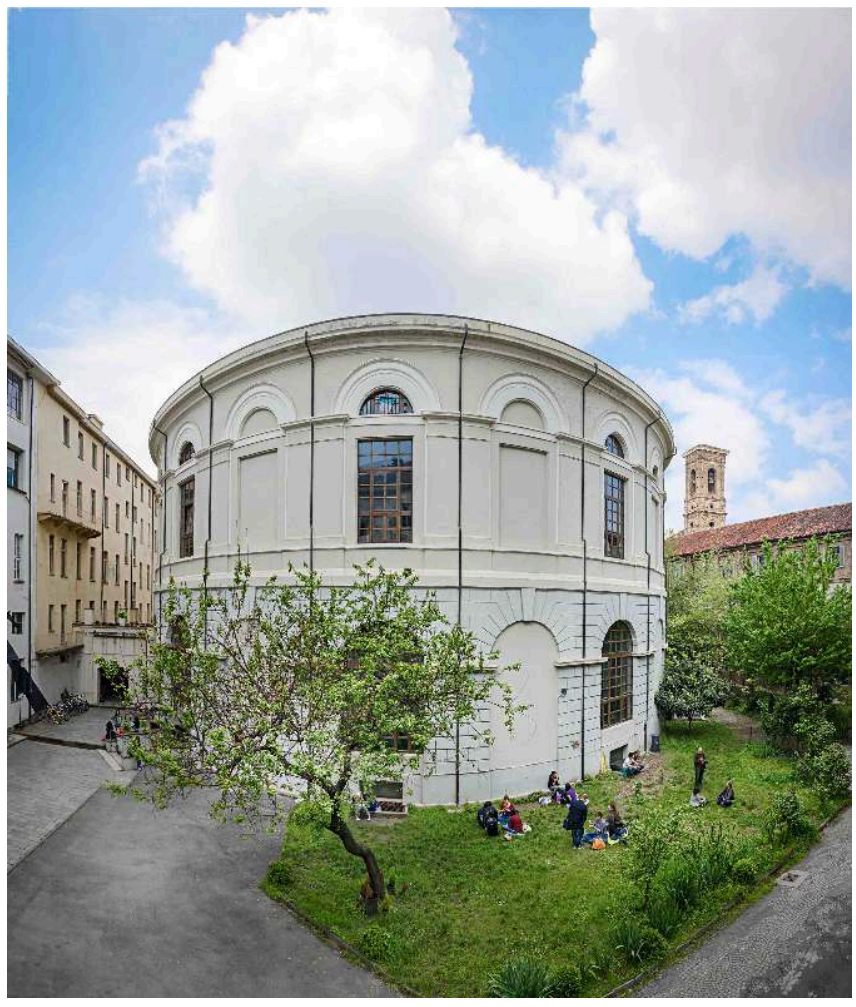

Accademia Albertina, "Rotonda".

(C) foto Fabio Amerio (Accademia Albertina di Belle Arti, Torino).

Fig. 4

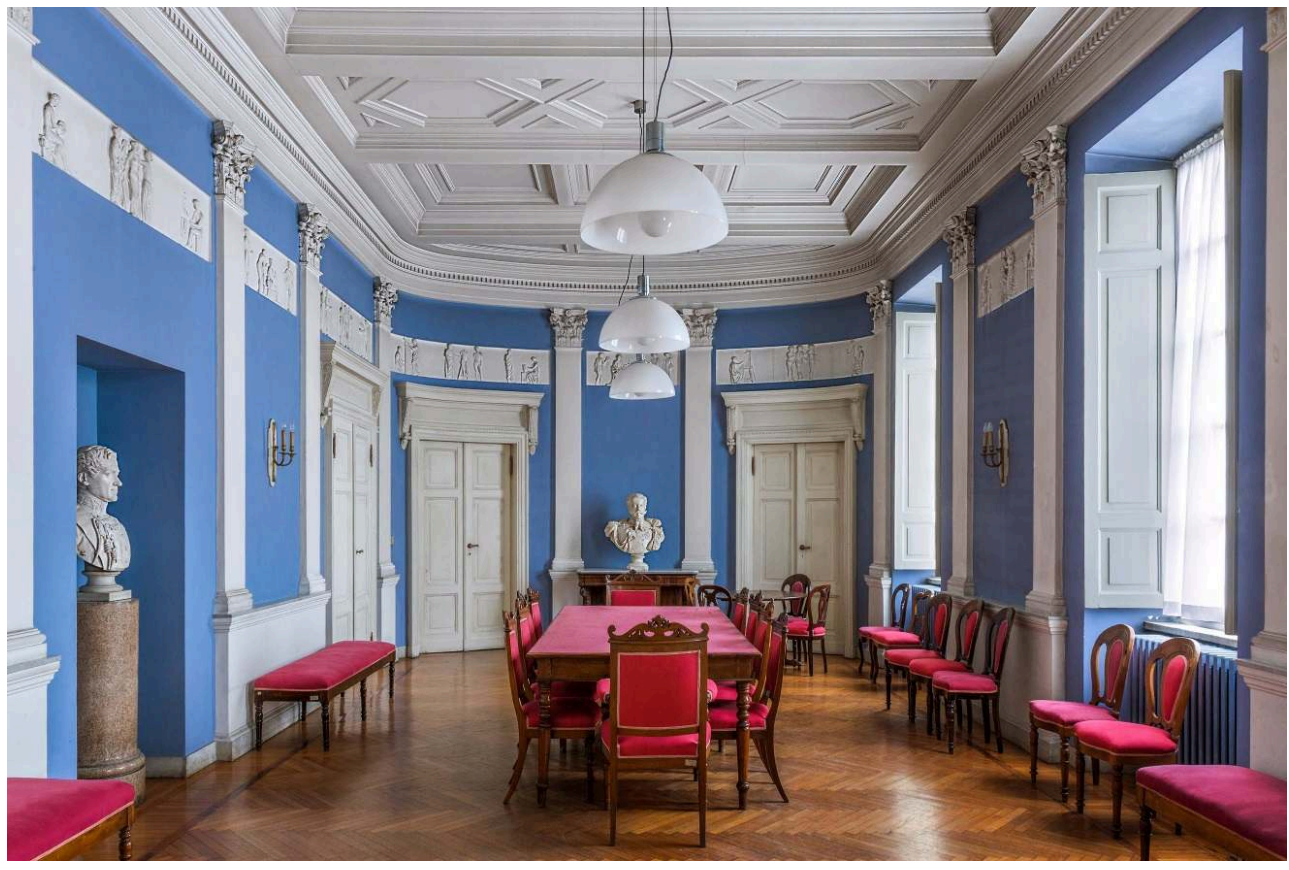

Accademia Albertina, sala con busto del re Carlo Alberto.

(c) foto Fabio Amerio (Accademia Albertina di Belle Arti, Torino). 
Fig. 5

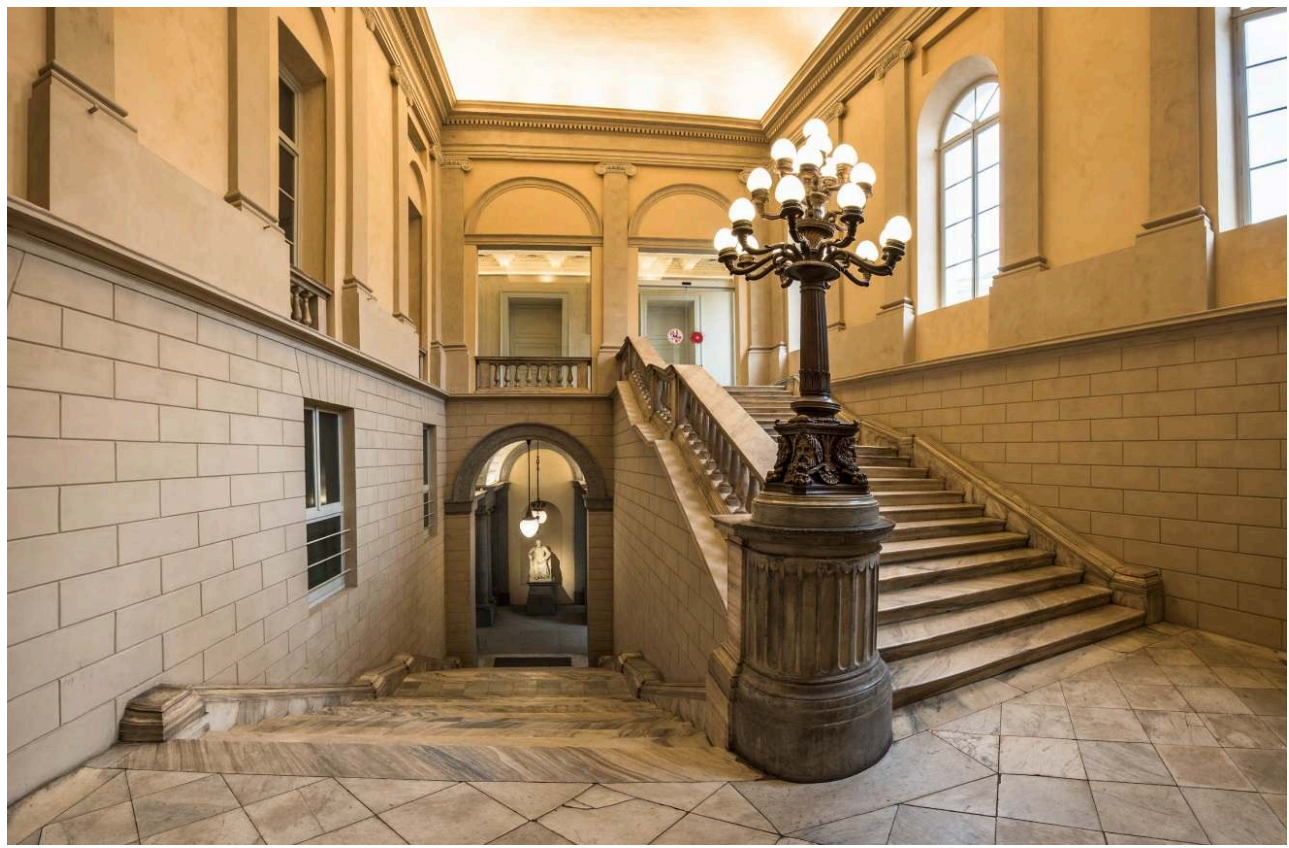

Accademia Albertina, scalone monumentale.

(c) foto Fabio Amerio (Accademia Albertina di Belle Arti, Torino).

L'Accademia è rifondata nel 1824 e resa autonoma dall'Università. Sono ammessi tra gli accademici i più importanti collezionisti e committenti di opere d'arte. All'epoca del re Carlo Alberto la collezione dei gessi dell'Accademia, divenuta "Albertina", è collocata nelle sale delle scuole di scultura, di anatomia e di nudo e raggiunge il numero di 850 opere come risulta dagli inventari del 1839, 1846 e 1856) ${ }^{12}$. Tali opere sono perdute in larga misura durante i bombardamenti della seconda Guerra Mondiale. Negli anni Trenta dell'ottocento, l'insegnamento d'anatomia è affidato a Francesco Bertinatti [fig. 6], i cui manoscritti redatti tra il 1837 e il 1839 sono conservati nella Biblioteca storica dell'Accademia [fig. ${ }^{\circ}$ ], insieme ai due volumi pubblicati dall'editore Marietti nel 1837 e alle addizioni, con 37 litografie, pubblicate in seguito fino al 1839, opere che sono presenti in vari esemplari per uso degli allievi ${ }^{13}$. 
Fig. 6

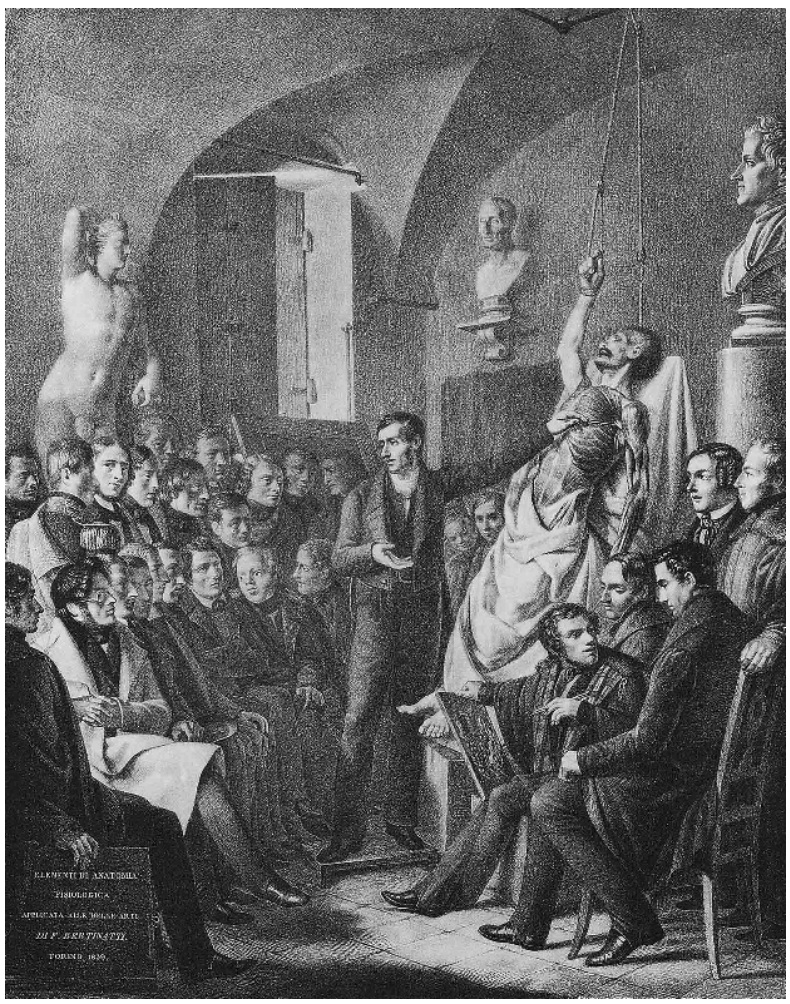

Corso d'anatomia, Paolo Emilio Morgari, litografia d'apertura dell'opera di BERTINATTI Francesco, Elementi di anatomia fisiologica applicata alle Belle Arti figurative, Torino, Pietro Marietti, 1837-1839.

Fig. 7

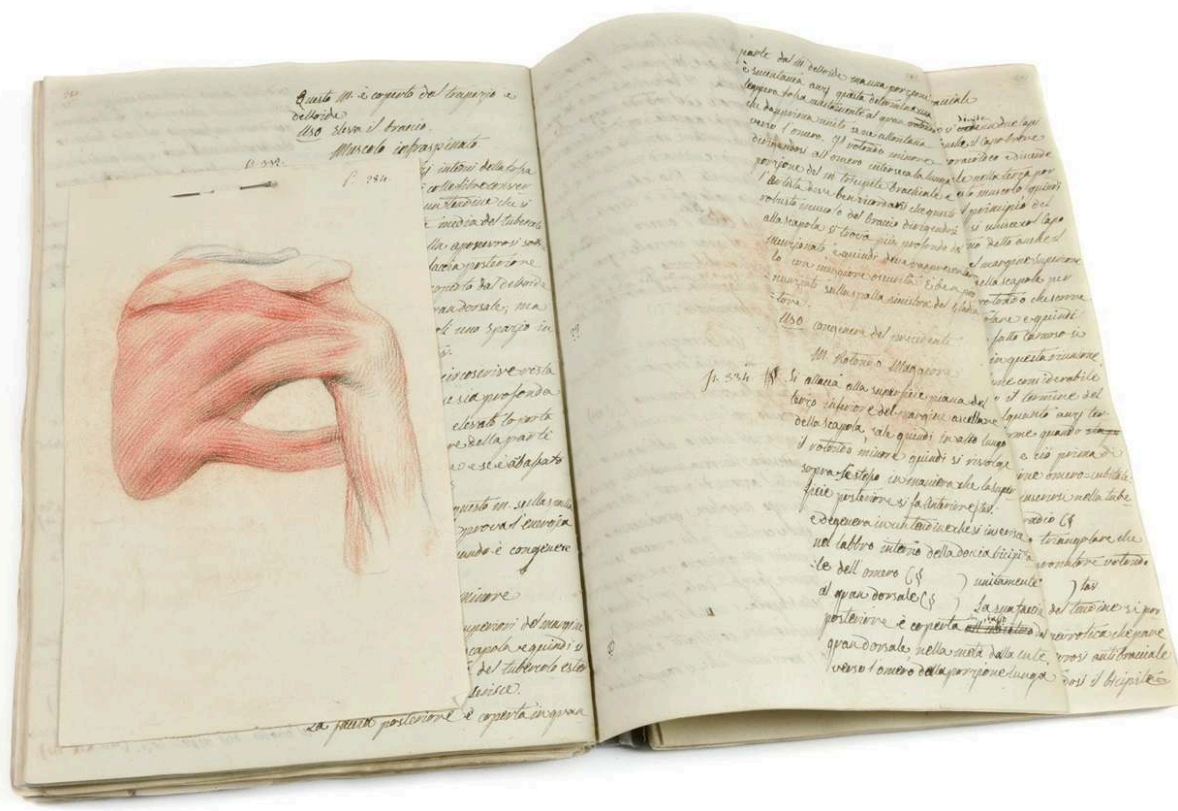

Muscoli della spalla, Francesco Bertinatti, pag. 284 del suo manoscritto d'anatomia conservato nella biblioteca dell'Accademia.

(c) foto Fabio Amerio (Accademia Albertina di Belle Arti, Torino) 
7 Una "Scuola d'ornato" è affidata a Pelagio Palagi per elevare il livello qualitativo dell'artigianato impiegato nei cantieri dei palazzi reali ${ }^{14}$. Il marchese Ferdinando di Breme nel 1849 è incaricato di rendere più libero ed efficace l'insegnamento. Egli s'ispira al modello delle botteghe medioevali, che comporta la possibilità per gli allievi di scegliere tra maestri in concorrenza tra di loro. Il vecchio corpo insegnante è quasi completamente sostituito da giovani di formazione internazionale, come Carlo Felice Biscarra, pittore e incisore nominato segretario nel 1860, e lo scultore svizzero Vincenzo Vela, tutti scelti dal di Breme ${ }^{15}$.

Una descrizione dettagliata dell'Accademia nel 1851 è offerta da Francesco Bertinaria ${ }^{16}$, che cita i modelli di architettura e i calchi di dettagli decorativi, i disegni e le incisioni di opere d'arte, le collezioni di opere d'arte, i sessanta cartoni di Gaudenzio Ferrari e altri pittori del Rinascimento donati dal re Carlo Alberto, gli acquerelli di Giuseppe Pietro Bagetti, i modelli di animali, gli scheletri, i quadri della collezione familiare donati dall'arcivescovo Vincenzo Mossi di Morano.

9 Negli anni Sessanta dell'Ottocento l'insegnamento della xilografia, funzionale alla produzione di immagini popolari e allo sviluppo delle edizioni d'arte, prende importanza in rapporto alla tradizionale incisione su rame ${ }^{17}$. La comparsa come materie di insegnamento della decorazione su porcellana e della piccola scultura in terracotta, testimonia dell'orientamento verso l'artigianato di qualità nella seconda metà del secolo $^{18}$. Nel 1872 una opera collettiva del maestro accademico Odoardo Tabacchi e dei suoi allievi celebra l'apertura del tunnel del Fréjus con un monumento munito di varie statue di grandi dimensioni ${ }^{19}$. Con un primo censimento regionale pubblicato nel 1878 , l'Accademia si interessa alla salvaguardia degli edifici e monumenti medioevali in pericolo. Il neo-medievalismo di fine secolo trova una testimonianza eclatante nella costruzione a Torino, in mezzo al parco del Valentino, per l'Esposizione generale italiana del 1884, di quel concentrato di copie d'arte e architettura piemontese e valdostana pre-rinascimentale, detto Borgo e Castello medioevale ${ }^{20}$.

10 A Torino, soltanto nel 1923, dopo una lunga resistenza in difesa di tradizioni locali, le classi preparatorie sono staccate dall'Accademia per dare origine a un istituto di belle arti, un liceo artistico uniforme alle analoghe scuole di tutta l'Italia ${ }^{21}$. Si trova una descrizione dei locali dell'Accademia e delle sue collezioni negli anni Venti nel libro di Luigi Cesare Bollea ${ }^{22}$. Nel 1941 iniziano il loro insegnamento in Accademia i pittori Felice Casorati ed Enrico Paulucci.

11 I bombardamenti della Seconda Guerra mondiale provocano gravi danni all'edificio e alle collezioni, in particolare quella dei gessi che è quasi cancellata. Tra le opere sfuggite al disastro si trova la copia della Porta del paradiso modellata nel 1425 da Lorenzo Ghiberti per il Battistero di Firenze [fig. 8]. 
Fig. 8

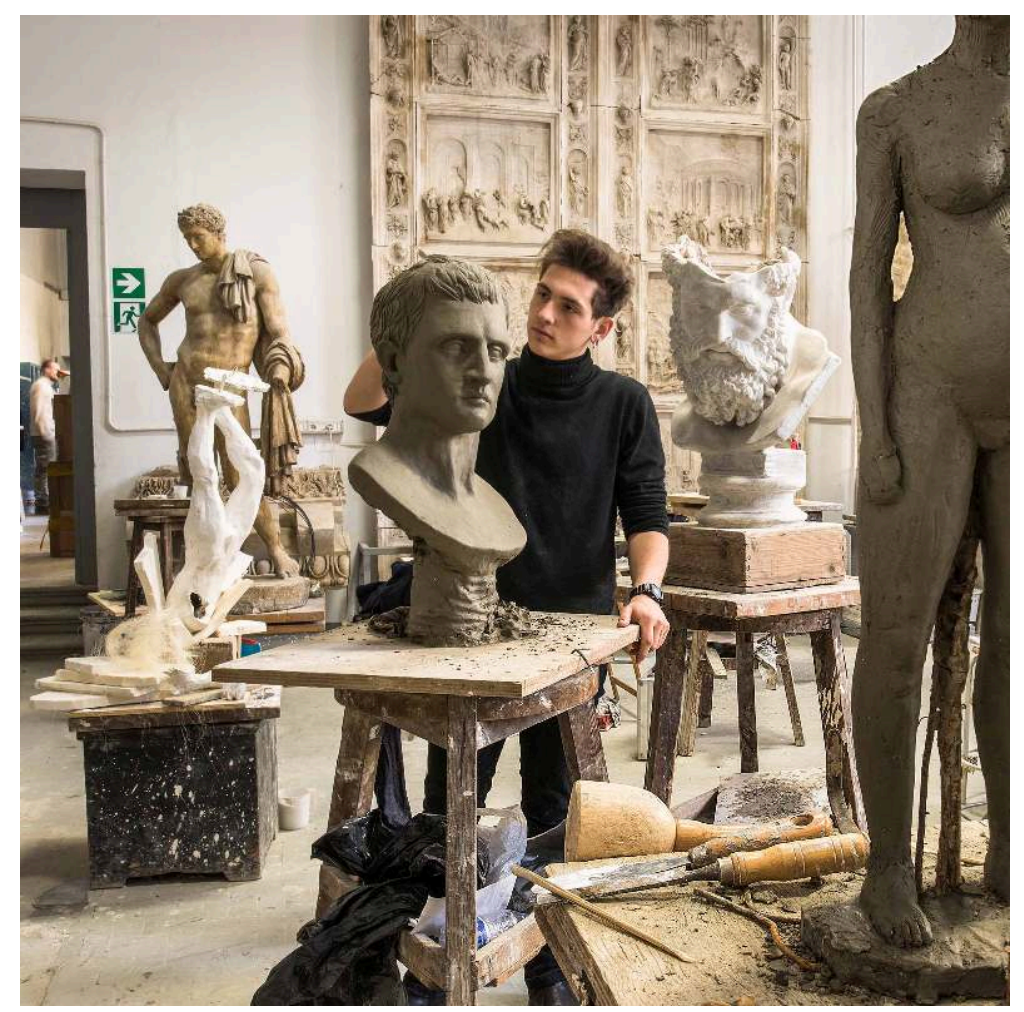

Accademia Albertina, atelier di scultura. Al muro calco della Porta del paradiso modellata nel 1425 da Lorenzo Ghiberti per il Battistero di Firenze.

(c) foto Fabio Amerio (Accademia Albertina di Belle Arti, Torino)

Dopo la Liberazione, si procede alla ricostruzione. L'edificio è restaurato, la pinacoteca è riorganizzata e riaperta. I lavori di riallestimento tecnologico della sala dedicata ai cartoni del Rinascimento terminano nel 2019. La Biblioteca e l'Archivio storico sono stati dotati recentemente di cataloghi ed inventari rinnovati. L'Accademia è oggi frequentata da allievi provenienti dal mondo intero.

\section{Le collezioni}

13 Le distruzioni della Seconda Guerra mondiale si sono sommate alla scarsa attenzione che per molto tempo è stata dedicata alla raccolta di opere prodotte dai maestri e dagli allievi dell'Accademia. Gli strumenti attuali della didattica comprendono gessi antichi e moderni (anatomia umana, statue dell'antichità classica, particolari decorativi), pitture, disegni, incisioni, fotografie, libri, manoscritti scientifici e artistici, mobili. Alcuni documenti dell'Archivio storico dell'Accademia sono testimonianze utilizzabili per l'insegnamento.

\section{La Biblioteca}

La biblioteca ${ }^{23}$ possiede un fondo antico che risale al Settecento e Ottocento, in maggioranza opere di storia, di letteratura, e raccolte di iconografia, senza trascurare le culture classica e religiosa, fonti inesauribili di soggetti per l'arte. Nel corso 
dell'Ottocento le opere accumulate sono sempre più eterogenee sia come riflesso delle nuove materie d'insegnamento, sia come risultato delle donazioni da parte di accademici e benefattori. Le acquisizioni riguardano le scienze della natura, il paesaggio, gli studi d'ingegneria, la medicina, l'anatomia, l'architettura, la decorazione, la storia antica e moderna, la letteratura. Ricerche sono in corso per ricostruire l'evoluzione della didattica artistica attraverso lo studio dei suoi strumenti ${ }^{24}$. Ci sono volumi che portano l'ex-libris di Laurent Pécheux che insegnava pittura e scultura nel 1778, altri vengono dalla donazione di Gerolamo Mattirolo (1847), dal fondo pervenuto a seguito della morte dell'architetto, ingegnere e professore Alessandro Antonelli (1888), dal fondo di Piero Alessandro Paravia (fine XIX secolo). L'acquisto di numerosi periodici è dovuto allo scultore e organizzatore culturale fascista Michele Guerrisi durante la seconda Guerra Mondiale ${ }^{25}$. Tra le opere acquisite all'epoca di Pécheux, si possono citare un Omero, le Metamorfosi di Ovidio, una Bibbia, un Trattato della pittura di Leonardo da Vinci unito ad una biografia a cura di Raphael Du Fresne (1786), libro che porta l'etichetta "ad uso degli allievi della Reale Accademia di pittura e scultura ${ }^{26 "}$. Il fondo bibliografico dell'Accademia per il periodo di Pécheux non manca di opere di storia orientale, greca e romana, con dettagli sulle consuetudini religiose, civili e militari (tra le opere etichettate dall'Accademia, quelle di Jean Baptiste Louis Crevier e Michel François Dandré-Bardon), opere biografiche in stile Vasari come quelle di Filippo Baldinucci (Notizie de' professori del disegno..., Torino, Piacenza, 1768 e anni seguenti), Bernardo De Dominici (Vite de' pittori, scultori et architetti napoletani, Napoli 1742 - 1743), Pellegrino Antonio Orlandi (Abecedario pittorico dei professori più illustri... Napoli 1775), Antonio Raffaello Mengs (Opere, pubblicate dal cavalier Niccola d'Azara, riviste da Carlo Fea e illustrate dal segretario dell'Accademia milanese di Brera, Roma, Pagliarini 1787) ${ }^{27}$.

\section{Gabinetto dei disegni e delle stampe}

15 Questo dipartimento ${ }^{28}$ ha quattro sezioni: l'antica conserva le opere dei secoli dal XVI al XVIII, la sezione moderna le opere del XIX secolo, la contemporanea quelle del $\mathrm{XX}^{\circ}$ secolo (vi si inseriranno le prove di concorso della scuola di incisione tra $1950 \mathrm{e}$ 1990), e la sezione delle donazioni ricevute in occasione delle grandi esposizioni. Tra i fondi speciali, alcuni sono dedicati alle opere prodotte dai membri della Società degli incisori all'acquaforte del XIX secolo, altri alle carte e mappe, ai fondi Casanova, Boselli e Poli. Il fondo del topografo paesaggista Giuseppe Pietro Bagetti, professore dell'Accademia, testimonia la sua partecipazione alle campagne napoleoniche in Italia. Si conservano le presse a braccio e a leva (per la stampa di incisioni, litografie e composizioni tipografiche) fabbricate a Torino da Bollito e Torchio tra il 1860 e il 1870, e a Monza da Amos dell'Orto nel 1864. Queste presse sono utilizzate per la scuola di incisione dalla seconda metà del XIX secolo fino ad oggi. Dal 1864 fino al 1888, la xilografia è insegnata dallo svizzero Giuseppe Salvioni, che l'Accademia invia a Parigi nel 1867 per rendersi conto delle innovazioni tecniche mostrate all'esposizione universale in materia di incisione ${ }^{29}$.

Le due sezioni del fondo fotografico, l'antica e la moderna, comprendono una serie importnate di fotoincisioni dell'inizio del $\mathrm{XX}^{\circ}$ secolo, ad uso degli allievi ${ }^{30}$. Il patrimonio video, formato dai video d'artista e dalla documentazione di spettacoli di teatro e balletto, è consultabile dagli allievi ${ }^{31}$. 


\section{La Pinacoteca}

Questa collezione, ${ }^{32}$ che risale al 1837, è tra i gioielli dell'Accademia. Le sue dodici sale, dove possono esercitarsi i copisti, sono anche aperte al pubblico [fig. ${ }^{\circ}$ ]]. Due sono $i$ fondi principali. La donazione, effettuata da monsignor Vincenzo Mossi di Morano nel 1828 , di duecento quadri a partire dal $\mathrm{XV}^{\circ}$ secolo permette l'esposizione, tra l'altro, di opere di Filippo Lippi, Domenico Puligo, Francesco Salviati, Maarten van Heemskerck, Defendente Ferrari, Bartolomeo Cavarozzi, Domenico e Anton Maria Piola, Gregorio De Ferrari, Giovanni Battista Carlone. Le dodici vedute di Venezia nel XVIII ${ }^{\circ}$ secolo sono di autore ignoto.

Fig. 9

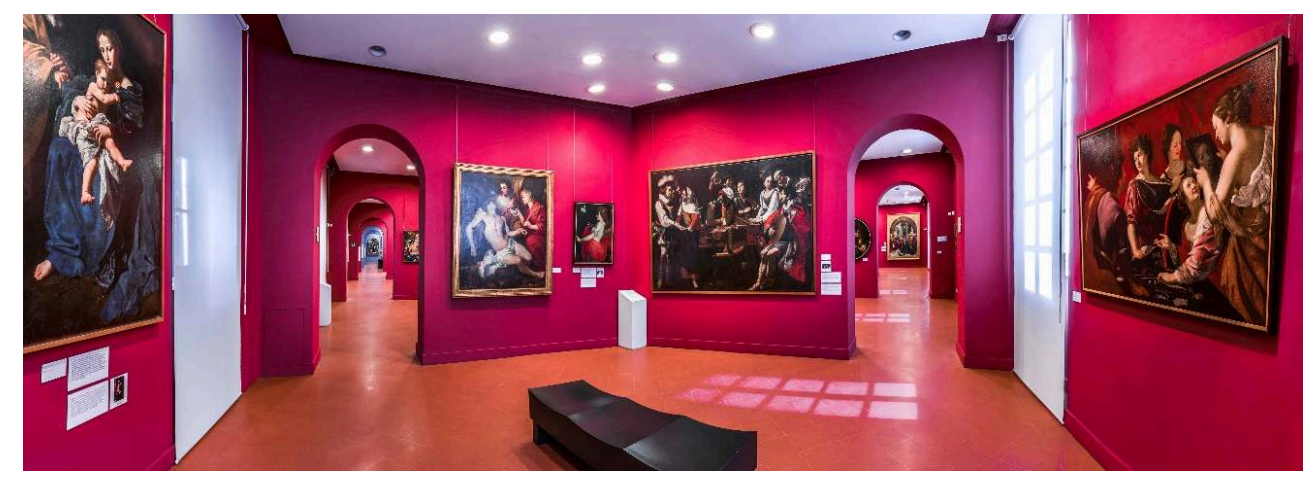

Accademia Albertina, sale della pinacoteca.

(c) foto Fabio Amerio (Accademia Albertina di Belle Arti, Torino).

Una sala specialmente attrezzata e illuminata, è oggi dedicata alla donazione da parte del re Carlo Alberto nel 1832 di sessanta opere datate tra il 1515 e il 1610, provenienti dall'atelier di Gaudenzio Ferrari e dei suoi allievi, con cartoni e disegni preparatori di quadri del Rinascimento [fig. ${ }^{\circ} 10$ ]. 
Fig. 10

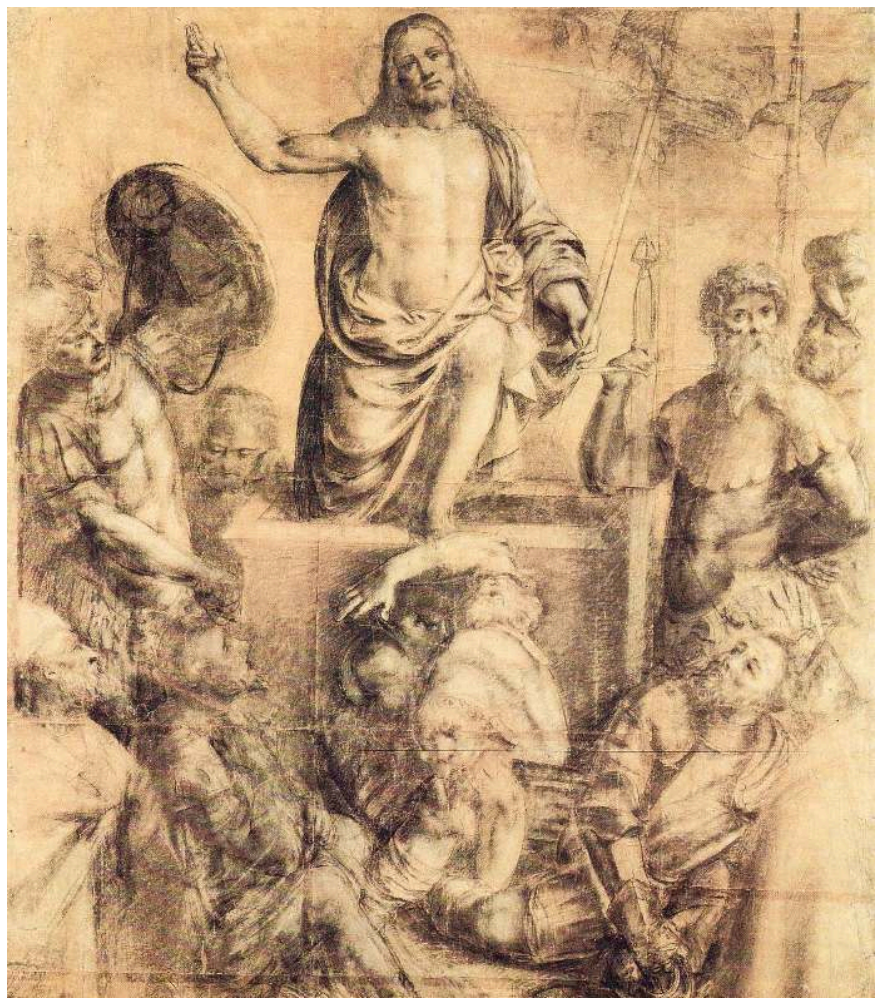

Resurrezione di Cristo e due santi vescovi, Giuseppe Giovenone il Giovane, 1550, cartone n. 342 della collezione dell'Accademia, disegno preparatorio del quadro del medesimo soggetto alla Galleria Sabauda di Torino.

Riproduzione Fabio Amerio (Accademia Albertina di Belle Arti, Torino).

19 Altre donazioni, più piccole ma assai importanti, sono quelle di Giovanni Volpato, insegnante dell'Accademia, che dona nel 1835 il quadro Nudo maschile, opera di JacquesLouis David e dei suoi allievi [fig. ${ }^{\circ} 11$ ], del pittore Giuseppe Monticoni che dona nel 1837 la sua collezione di quadri del XVII'secolo, della vedova del pittore Giuseppe Pietro Bagetti (1842) che dona anche il bosto in marmo di suo marito fatto da Giacomo Spalla, e una serie di opere degli scultori Ignazio et Filippo Collino, chiamati a insegnare all'Accademia dopo la riforma del 1778. 
Fig. 11

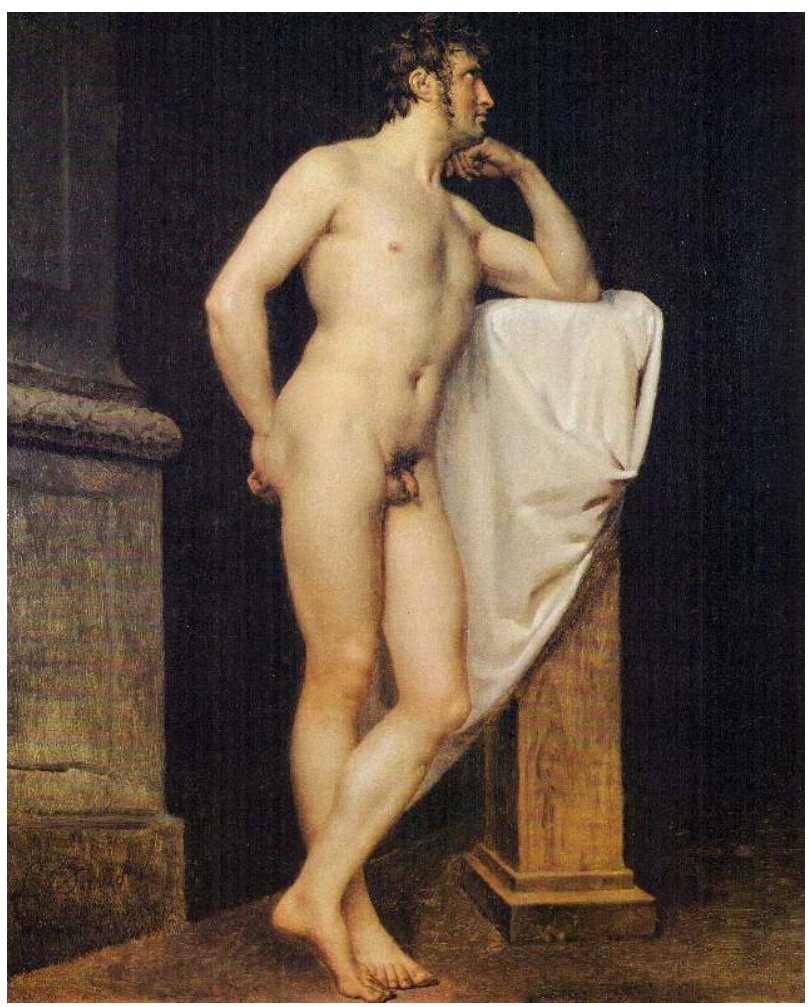

Nudo accademico, Jacques-Louis David (studio di), olio su tela, Pinacoteca dell'Accademia. Riproduzione Fabio Amerio (Accademia Albertina di Belle Arti, Torino).

20 La collezione di pitture di Giacomo Grosso (insegnante tra il 1906 e il 1933) deriva da un acquisto dello Stato nel 1939 e comprende il quadro La nuda [fig. 12], oggi in prestito alla Galleria d'arte moderna di Torino. I fondi del XX secolo concernono numerosi artisti, tra i quali si possono citare anche Carlo Bonatto Minella, Angelo Beccaria, Bartolomeo Ardy, Ludovico Raymond, Luigi Mainolfi, Luigi Stoisa, Paolo Grassino, Carlo Oreste Strocco. Le opere conservate nei depositi, montate su griglie scorrevoli, sono visibili su richiesta. 
Fig. 12

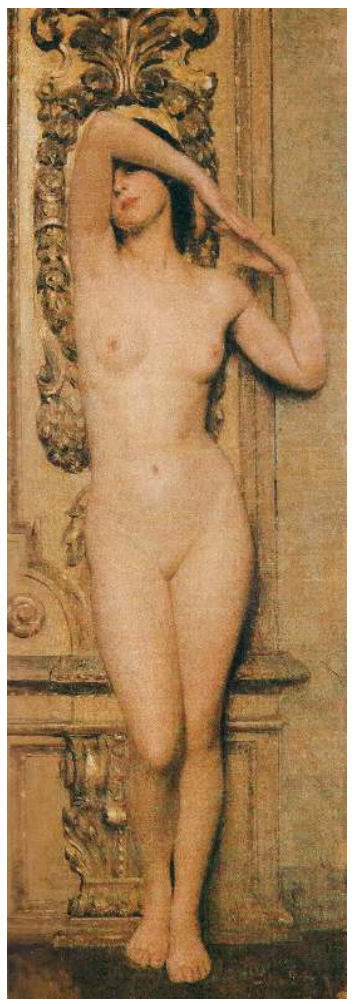

La Nuda, Giacomo Grosso, olio su tela, deposito dell'Accademia alla Galleria d'arte moderna di Torino. Riproduzione Fabio Amerio (Accademia Albertina di Belle Arti, Torino).

21 I busti di vari personaggi importanti per la storia dell'Accademia sono esposti nelle sale di rappresentanza. Nell'atrio d'ingresso si trova la statua monumentale dell'arcivescovo Mossi di Morano, opera dello scultore Carlo Marocchetti ${ }^{33}$.

\section{La gipsoteaca}

Dalla riforma del 1778, l'Accademia accumula una vasta collezione di copie di grandi dimensioni di statue greche e romane, a partire da quelle commissionate a Roma da Laurent Pécheux ${ }^{34}$. Tra le opere sopravvissute ai bombardamenti dell'ultima guerra, si possono citare la Nike di Samotracia e il Gladiatore combattente, che è utilizzato per lo studio del sistema muscolare [fig. 13] ${ }^{35}$. Acquisizioni del XIX secolo concernono, tra l'altro, lo scultore Antonio Canova (che dona il Torso di Fidia) e Bertel Thorvaldsen che dona la statua di Psiche. Le acquisizioni più numerose sono quelle dovute a Luigi Canina (1843), Giovanni Battista Biscarra (1845), Crescentino Caselli (1894). Occorre tuttavia notare che l'utilizzo frequente delle opere per la didattica pone dei problemi dal punto di vista della conservazione e della fruibilità museale per il grande pubblico. 


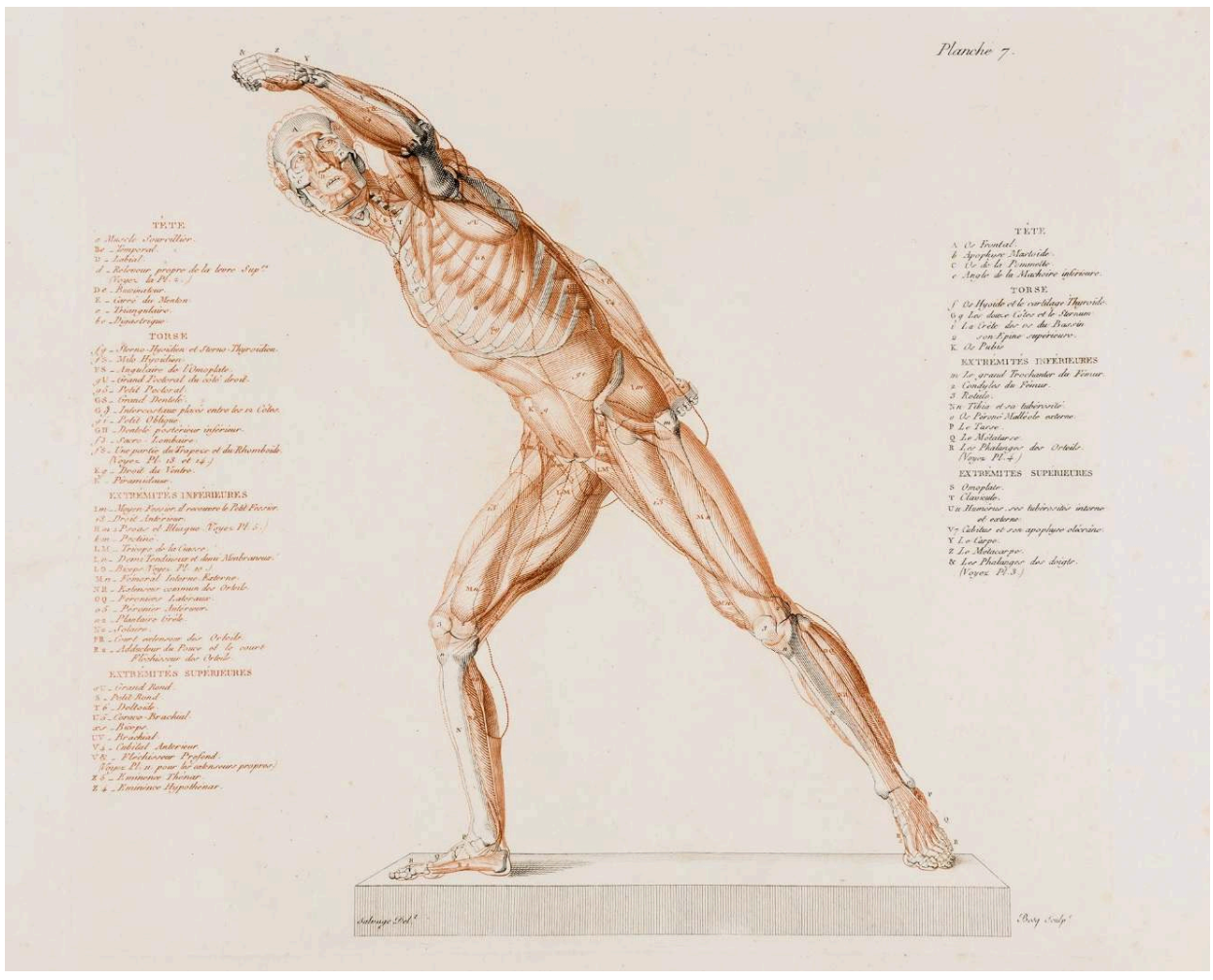

Anatomie du gladiateur combattant, disegno di Jean-Galbert Salvage (il dottore militare di Napoleone), Litografia di Jean Bosq, piatto n. 7 da un portfolio pubblicato a Parigi, s. d., Biblioteca dell'Accademia. Riproduzione Fabio Amerio (Accademia Albertina di Belle Arti, Torino).

\section{NOTE}

1. DALMASSO Franca, «L'Accademia Albertina: storia e artisti », in DALMASSO Franca, GAGLIA Pierluigi \& POLI Francesco (dir.), L'Accademia Albertina di Torino, Torino, Arti grafiche, 1982, p. 11. 2. ROBERTO Maria Teresa, «Breve storia dell'Accademia », in CASSESE Giovanna (dir.), Accademie patrimoni di Belle Arti. L'Accademia Albertina di Torino, Roma, Gangemi, 2013, p. 109 ss.

3. BOLLEA Luigi Cesare, Gli storici dell'Accademia, Torino, Bocca, 1930, p. 45.

4. ROBERTO Maria Teresa, cit., p. 109.

5. LALANDE Joseph-Jérôme Lefrançois de, Voyage en Italie, Paris, Veuve Desaint, 1786, p. 245- 247.

6. DALMASSO Franca, cit., p. 24.

7. DALMASSO Franca, cit., p. 22-28.

8. ROBERTO Maria Teresa, cit.

9. POLI Francesco, "La sede dell'Accademia di Belle Arti: l'attuale edificio, precedenti collocazioni, progetti di altre sistemazioni”, in DALMASSO Franca, GAGLIA Pierluigi \& POLI Francesco (dir.), L'Accademia Albertina di Torino, Turin, Arti grafiche, 1982, p. 89 ss.

10. DALMASSO Franca, cit., p. 35. 
11. GRASSI Rosella, «Il patrimonio storico-artistico. Breve storia della struttura e degli spazi più rappresentativi ", in CASSESE Giovanna (dir.), Accademie patrimoni di Belle Arti. L'Accademia Albertina di Torino, Roma, Gangemi, 2013, p. 112 ss.

12. DALMASSO Franca, cit., p. 34.

13. BERTINATTI Francesco, Elementi di anatomia fisiologica applicata alle Belle Arti figurative, Torino, Pietro Marietti, 1837-1839. Cfr. MUSIARI Antonio, «Biblioteconomia centripeta. Il nucleo originario degli acquisti di trattati anatomici e fogli a stampa ", in MERLINO Roberta, MUSIARI Antonio, TARTAGLINO Fernanda (dir.), Corpi di carta. Testi e immagini di anatomia nella Biblioteca storica dell'Accademia Albertina. Catalogo della mostra (Torino, 1 luglio-8 settembre 2017), Torino, Albertina Press, 2017, p. 13.

14. DALMASSO Franca, cit., p. 35.

15. DALMASSO Franca, cit., p. 39.

16. BERTINARIA Francesco, "Una visita alla Reale Accademia Albertina di Belle Arti », Gazzetta piemontese, 21 agosto 1851 .

17. DALMASSO Franca, cit., p. 46.

18. DALMASSO Franca, cit., p. 51.

19. DALMASSO Franca, cit., p. 58.

20. DALMASSO Franca, cit., ibidem.

21. DALMASSO Franca, cit., p. 70-72.

22. BOLLEA Luigi Cesare, La Regia Accademia Albertina delle belle arti e la Regia casa di Savoia, Torino, Bocca 1930.

23. POLI Francesco, «La biblioteca: testi didattici, stampe, disegni originali », in DALMASSO Franca, GAGLIA Pierluigi \& POLI Francesco (dir.), L'Accademia Albertina di Torino, Torino, Arti grafiche, 1982, p. 193 ss.

24. GRASSI Rosella, «La Biblioteca storica », in CASSESE Giovanna (dir.), Accademie patrimoni di Belle Arti. L'Accademia Albertina di Torino, Rome, Gangemi, 2013, p. 115, segnala che F. Cafagna studia la scuola di anatomia e B. Zanelli la scuola di architettura.

25. GRASSI Rosella, ibidem. Cfr. anche Archivio storico dell'Accademia Albertina, TO 22 (48) et TO 84.

26. GRASSI Rosella, cit., p. 116. Si tratta di un complesso di libri identificato da Francesco Poli sulla base della lista trascritta da Luigi Cesare Bollea nel 1936.

27. GRASSI Rosella, cit, ibidem.

28. GRASSI Rosella, «Il Gabinetto dei disegni e stampe », in CASSESE Giovanna (dir.), Accademie patrimoni di Belle Arti. L'Accademia Albertina di Torino, Roma, Gangemi, 2013, p. 118-120.

29. SALVIONI Giuseppe, Cenni storici sulla zilografia, ossia incisioni in legno, seguiti da alcune considerazioni intorno alle attuali condizioni di quest'arte in Italia, Torino, Civelli 1868 ; citato da FANELLI Franco, «Il patrimonio storico del corso di tecniche dell'incisione e grafica d'arte », in CASSESE Giovanna (dir.), Accademie patrimoni di Belle Arti. L'Accademia Albertina di Torino, Roma, Gangemi, 2013, p. 122.

30. GRASSI Rosella, « Il Gabinetto dei disegni e stampe », cit., p. 118.

31. AUSONI Alberto, «La videoteca », in CASSESE Giovanna (dir.), Accademie patrimoni di Belle Arti. L'Accademia Albertina di Torino, Roma, Gangemi, 2013, p. 124.

32. PETRUCCI Francesca, "La Pinacoteca Albertina », in CASSESE Giovanna (dir.), Accademie patrimoni di Belle Arti. L'Accademia Albertina di Torino, Roma, Gangemi, 2013, p. 124-126. Cfr. anche GAGLIA Pierluigi, "La formazione della pinacoteca », in DALMASSO Franca, GAGLIA Pierluigi \& POLI Francesco (dir.), L'Accademia Albertina di Torino, Torino, Arti grafiche, 1982, p. 125 ss.

33. PETRUCCI Francesca, cit., p. 126.

34. GRASSI Rosella \& ZANELLI Beatrice, «La Gipsoteca », in CASSESE Giovanna (dir.), Accademie patrimoni di Belle Arti. L'Accademia Albertina di Torino, Roma, Gangemi, 2013, p. 128. 
35. La litografia, incisa da Bosq, che mostra i muscoli del gladiatore, è derivata da un disegno del medico militare di Napoleone, Jean Galbert Salvage, Anatomie du gladiateur combattant, planche n.7, Paris, s.d.

\section{AUTORE}

\section{MARCO CARASSI}

marco.carassi47@gmail.com 